\title{
Identification and tissue distribution of mRNAs encoding salmon-type calcitonins-IV and $-V$ in the rainbow trout
}

\author{
Yoshie Hidaka and Masakazu Suzuki \\ Department of Biology, Faculty of Science, Shizuoka University, Ohya 836, Shizuoka City, Shizuoka 422-8529, Japan \\ (Requests for offprints should be addressed to Masakazu Suzuki; Email: sbmsuzu @ipc.shizuoka.ac.jp)
}

\begin{abstract}
Four types of calcitonin are produced in salmonid fish, although their functional diversity is almost unknown. To explore the significance of these isoforms, we have characterized salmon-type calcitonin (sCT) mRNAs in the rainbow trout (Oncorhynchus mykiss), and examined their tissue distribution. In addition to the previously isolated SCT-I CDNAs, two new forms of SCT CDNA were cloned from the ultimobranchial gland, and one of them (SCT-IV CDNA) was predicted to encode an N-terminal peptide of 80 amino acid residues, a putative cleavage site Lys-Arg, SCT-IV, a cleavage and amidation sequence Gly-Lys-Lys-Arg, and a C-terminal peptide of 18 amino acids. The SCT-IV precursor was $78 \%$ identical with the rainbow trout SCT-I precursors. The other cloned CDNA encoded a precursor for a novel CT, SCT-V. The SCT-V peptide was different from SCT-IV by only one amino acid residue: Val at position 8 in the latter was replaced by Met. The SCT-V precursor had 80 and $90 \%$ identity with the SCT-I and -IV precursors respectively. No cDNA clones were obtained for SCTs-II or -III.

Tissue distribution of SCT-I, -IV and -V mRNAs was examined by RT-PCR and specific cleavage with restriction enzymes. An amplified fragment from sCT-I mRNA was detected not only in the ultimobranchial gland, but also in the gills, testis and ovary. RT-PCR analysis coupled to restriction digestion further revealed that SCT-IV mRNA was expressed in both the testis and the ultimobranchial gland. The expression sites of SCT-IV mRNA were localized to the Leydig cells of the testis and to the parenchymal cells of the ultimobranchial gland, by in situ hybridization histochemistry. Although the amino acid sequence of SCT-V peptide was nearly the same as that of SCT-IV, the SCT-V gene showed a much wider pattern of expression: the band amplified by RT-PCR was detected in all the tissues examined except the kidney, gills and blood cells. The SCT-V mRNA was shown to be localized in the parenchymal cells of the ultimobranchial gland, but not in other tissues at the cellular level, suggesting very low expression of SCT-V mRNA in those tissues. Our results show different patterns of tissue expression of three types of $S C T$ genes in the rainbow trout, suggesting that SCTs-I, -IV and $-\mathrm{V}$ might differ in their local actions.
\end{abstract}

Journal of Molecular Endocrinology (2004) 32, 963-974

\section{Introduction}

Calcitonin (CT) was initially discovered as a hypocalcaemic hormone in mammals. It is abundantly produced by the parafollicular cells of the thyroid gland, and principally inhibits osteoclast-mediated bone resorption (Sexton et al. 1999). It is also reported that CT is secreted by a variety of extrathyroidal tissues (Becker et al. 1979, Ding et al. 1994), and that CT receptor mRNA is expressed at many of these sites, such as the brain (Sheward et al. 1994), kidney, lung and stomach (Kuestner et al. 1994), suggesting diverse local actions of CT.

Down the phylogenetic lineages of vertebrates, non-mammalian calcitonin is produced by the ultimobranchial gland, and the sequences of CT peptides and mRNAs have been determined in a number of species, from fish to mammals (Sasayama 1999, Suzuki et al. 1999b). Sequence analysis reveals that CT is a 32 amino acid polypeptide that is derived from a larger precursor molecule, and further that some species produce 
more than one form of CT (Suzuki et al. 1999b). Within the Salmonidae, four types of CT, sCTs-I to -IV, have been reported to date, and it has been suggested that pink salmon could express sCTs-I, -II and -IV (Jansz et al. 1996). Jansz et al. (1996) also suggested that sockeye and chum salmon possess both sCT-I and sCT-II, whereas coho salmon has sCTs-II and -III. It has also been suggested that, in fish, CT might control the uptake of dietary calcium from the digestive tract (Suzuki et al. 1999a), suppress osteoclastic activities in the scales (Suzuki et al. 2000a), and be involved in reproductive physiology (Bjornsson et al. 1986, Maubras et al. 1990). However, little is known about differences between the physiological roles of the isoforms of CT existing in the same species. Investigation of the tissue distribution of the isohormones is a prerequisite for the understanding of their functional differences. In the present study, we have cloned and sequenced cDNAs encoding two types of ${ }_{S C T}$ (IV and V) from the ultimobranchial gland of rainbow trout, and compared the tissue distribution of their mRNAs with that of sCT-I mRNA.

\section{Materials and methods}

\section{Animals and sampling}

Two-year-old rainbow trout (Oncorhynchus mykiss) of both sexes were collected at Fuji Trout Hatchery, Shizuoka, Japan, in March and May. Ultimobranchial glands and other organs were carefully dissected out, and blood cells were collected by centrifuging the blood at $3000 \boldsymbol{g}$ for $4 \mathrm{~min}$. The samples were immediately frozen in liquid nitrogen, and stored at $-80{ }^{\circ} \mathrm{C}$ in a deep-freezer. In addition, various tissues of adult trout were fixed in $4 \%$ paraformaldehyde at $4{ }^{\circ} \mathrm{C}$ overnight for histological analysis. Adult rainbow trout of a different strain were obtained from a commercial dealer, Yabe Trout Hatchery, Tokyo, Japan, in January. Wistar rats of 6 weeks of age (Japan SLC, Shizuoka, Japan) were anaesthetized with ether, and dissected to provide tissues for RT-PCR analysis.

\section{Construction of cDNA library and DNA cloning}

Total RNA was extracted from 120 ultimobranchial glands of the trout using TRIzoL reagent
(Gibco BRL, Rockville, MD, USA), and poly(A) ${ }^{+}$ RNA was prepared with oligo-dT-coated latex beads (Oligotex-dT30 super; Takara, Kyoto, Japan), according to the manufacturer's instructions. cDNA was prepared with a ZAP Express cDNA synthesis kit (Stratagene, La Jolla, CA, USA), and then a cDNA library was constructed using a ZAP Express cDNA Gigapack III Gold cloning kit (Stratagene). The resultant cDNA library contained $7 \cdot 0 \times 10^{6}$ recombinant lambda phages, and was amplified to $7 \cdot 6 \times 10^{8}$ plaqueforming units $(\mathrm{pfu}) / \mathrm{ml}$.

\section{Preparation of cDNA probe}

$\operatorname{Poly}(\mathrm{A})^{+}$RNA $(0.5 \mu \mathrm{g})$ from the ultimobranchial gland was reverse-transcribed in $20 \mu \mathrm{l}$ buffer containing 80 pmol oligo-dT19 primer, $1 \mathrm{mM}$ dNTP, 20 units RNase inhibitor (Toyobo, Osaka, Japan), and 10 units Rous-associated virus 2 (RAV-2) reverse transcriptase (Takara), essentially as described previously (Suzuki et al. 1992). PGR was subsequently carried out with the sCT primers (Table 1) using a Program Temp Control System, PG-701 (Astec, Fukuoka, Japan). The amplification profile for 30 cycles was: dissociation at $94{ }^{\circ} \mathrm{C}$ for $1.5 \mathrm{~min}$, annealing at $55^{\circ} \mathrm{C}$ for $1.5 \mathrm{~min}$ and extension at $72{ }^{\circ} \mathrm{C}$ for $2.5 \mathrm{~min}$. The final cycle included polymerization for $8 \mathrm{~min}$. Amplification products were separated by electrophoresis and a major band was subcloned into pGEM-3Z vector (Promega, Madison, WI, USA). Sequencing reactions were conducted with a thermo sequenase cycle sequencing kit (USB, Gleveland, OH, USA), and nucleotide sequences were analysed using a Li-Cor automated DNA sequencer, model 4200L-2G (Li-Cor, Lincoln, NE, USA). A DNA probe was synthesized from a cDNA fragment encoding sCT-I, using a digoxigenin (DIG)-high prime kit (Roche Diagnostics GmbH, Mannheim, Germany).

\section{DNA cloning and sequence analysis}

Approximately 18000 recombinants from the amplified cDNA library were screened by plaque hybridization. Hybridization with the above cDNA probe and post-hybridization washing were performed essentially according to the manufacturer's instructions. Hybridization signals were detected with $25 \mathrm{mM}$ CSPD, a 1,2-dioxetane chemiluminescent enzyme substrate (Tropix, Bedford, MA, 
Table 1 The nucleotide sequences of PCR primers

\begin{tabular}{|c|c|c|}
\hline Primer & & Sequence \\
\hline sCT & Sense & 5'-CAAGCGTTGCTCCAACCTCA-3' \\
\hline sCT & Antisense & 5'-CGTTTCTTGCCAGGCGTGCC-3' \\
\hline rtCT-I & Sense & 5'-AATCTGAGCGGTCCTCCCCT-3' \\
\hline rtCT-I & Antisense & 5'-TCAAAAAGGGGGATTTTAAC-3' \\
\hline rtCT-IV & Sense & 5'-ATTATAACGTCCTCCTGGTT-3' \\
\hline rtCT-IV & Antisense & 5'-GAAGAAACTATTTATTATGGTTG-3' \\
\hline rtCT-V & Sense & 5'-СТСТTTTTСАСТTTTААТTTТAAT-3' \\
\hline rtCT-V & Antisense & 5'-GTCATTTTTAGATAAGTTCTC-3' \\
\hline $\mathrm{mCT}$ & Sense & 5'-TGAGGGCTCTAGCTTGGACA-3' \\
\hline $\mathrm{mCT}$ & Antisense & 5'-TGGTGGTTTGTCTCCAAGTC-3' \\
\hline sEF1- $\alpha$ & Sense & 5'-GGCTGGTTCAAGGGATGGAA-3' \\
\hline$s E F 1-\alpha$ & Antisense & 5'-ATTGGAGGGTCGTTCTTGCT-3' \\
\hline
\end{tabular}

USA), on Hyperfilm-ECL film (Amersham Biosciences, Piscataway, NJ, USA) after incubation with alkaline phosphatase-conjugated anti-DIG antibody (Roche). The pBK-GMV phagemid vectors with inserts were excised in vivo from the ZAP express vectors of positive recombinants, using the ExAssist helper phage (Stratagene). The nucleotide sequences of these DNAs were analysed using a Li-Cor automated DNA sequencer. A phylogenetic tree of CT precursors from the rainbow trout, chum salmon (Poeschl et al. 1987), puffer fish Fugu (Clark et al. 2002), chicken (Lasmoles et al. 1985), mouse (Rehli et al. 1996), rat (Jacobs et al. 1981) and human (Graig et al. 1982) was constructed on the basis of the amino acid sequence, using the Clustal W algorithm (Thompson et al. 1994).

\section{Tissue distribution of CT mRNAs}

Total RNA was extracted from various tissues of rainbow trout, using TRIzol reagent (Roche). After treatment with deoxyribonuclease I (Takara), $10 \mu \mathrm{g}$ total RNA were subjected to RT-PCR, as described above. Preparation of total RNA from rat tissues and its RT-PCR analysis were conducted similarly with the mCT primers (Table 1). As for the trout, a pair of specific primers were synthesized and used for detection of each of sCT-I, -IV and -V mRNAs (Table 1). The sCT-I primers were designed to recognize the sequences common to rainbow trout cDNAs for $\mathrm{sCT}$ s-Ia and -Ib (accession numbers AB094135 and AB094136 respectively; Hidaka et al. 2004), and the PCR amplification specific to each of sCT-I and -IV
mRNAs was confirmed by restriction enzyme cleavage: PstI (Takara) for the former, and NspI (Roche) for the latter. Elongation factor $1-\alpha(E F 1-\alpha)$ cDNA (BE669055) was also amplified with specific primers (Table 1), as an endogenous control. A $10 \mu \mathrm{l}$ aliquot of each amplified product was electrophoresed through an ethidium bromidestained 2\% agarose gel and photographed with a FAS-III digital camera system. Semiquantitative analysis was carried out by calculating the relative amount of each sCT amplicon to the corresponding EF1- $\alpha$ band, using Scion Image software.

\section{Localization of sCT-I mRNA}

In situ hybridization histochemistry was carried out on wax sections of the ultimobranchial glands. In situ hybridization of the tissue sections was performed as described previously (Suzuki et al. 1997). Antisense and sense RNA probes were prepared by in vitro transcription from the fragments of rainbow trout sCT-IV and -V cDNAs, using a DIG-RNA labelling kit (Roche).

\section{Results}

\section{Molecular cloning of SCT cDNA}

There was a distinct cDNA band of approximately $100 \mathrm{bp}$ observed after gel electrophoresis of RT-PCR products, which were amplified from the ultimobranchial gland mRNA using the sCT primers (Table 1). After isolation and subcloning of the cDNA fragment, the nucleotide sequences 
SCT-IV ---TGGTCTTCAAGTTGAGAGAAGTCAACTTAGGCGTTTCATATAAAGCTATATATTTTTTTTTTGCCCGTTGATTAACACGAAAGGGAACCATGGTTATG - 1

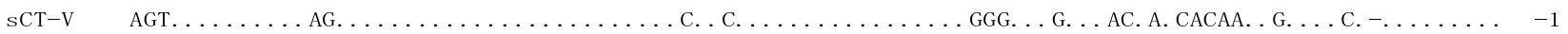

SCT-IV ATGGTTATGTTGAAGATTTCTGCTTTCCTTGTTGCTTATGCCCTGGTCATTTGCCAGATGTACAGCTCACAAGCAGCCCCATCCAGACCGGGCATAGAGT 100

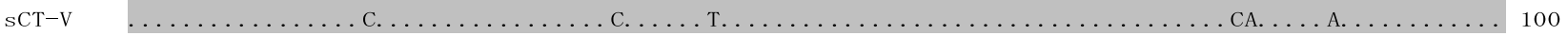

SCT-IV CCATGACAGACCGAGTCACCCTTACGGACTACGAGGCAAGAAGGTTACTCAACGCCATAGTCAAGGAGTTTGTGCAGATGACAGCCGAAGAGCTGGAGCA 200

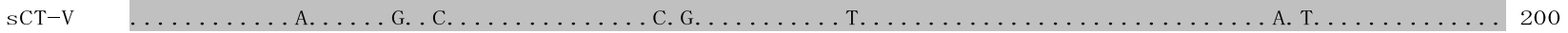

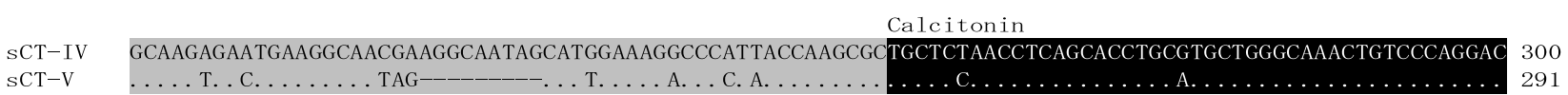

SCT-IV CTGCACAAATTACAAACGTTTCCCGCACGGACGTGGGCGCGGGCACGCCTGGCAAGAAACGCAGCGCGCCCGAGAGCGAACGCTATGCAAGCTACGGGA 400

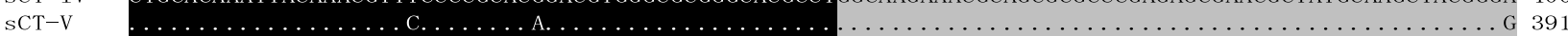

SCT-IV AGACATTTGACAGCATCTAACCCTCCGCCCTCCTCCTATTCCCTCTCTTTTCATTATAACGTCCTCCTGGTTTTTGTTTGACAAGTCTCAGTTGCATGTG 50O SCT $-\mathrm{V} \quad \ldots \ldots$. . . . . . . . . . T. CT. . GT. . . CC. GTTC. CAC. C. TT. . CACTTT. AA. TTGAA. G. CCTCC. . GTTTTAAATCTC. . . . . 491

SCT-IV GATTTTTCCTGCTTGATTGACCTAAGAGCGAATCTCCCATTGCAACCCCCACTTCTTCATTTTTGTATTCTTTTGACA-ATTCCTTTCCGTTTTCGGCAA 599 SCT-V … . G. . . . . . CATTAGA. CAGATTTCC---. . C. . . . G. . C. G. GACTT. . CCC. TC. TC. . . TGATA. . . . . C. . CC. . . . . . G 587

SCT-IV AGAATGAATGACATGTTGTATCTGTTCAGACAATCAACTCTTCTAAATTGCAATTATTCAAAAGAATGAATCAACATATATCGATTTTCTTTCAGTGTGT 699 sCT-V .... G. . CGTTT. GACAA. CGAAA. GAGA. . TTATCTAAAA----. TGACA. T. AT. -. . . . . . . . . . G. TG. . . GATG. . . T. . T. TGAGTG 682

SCT-IV AT-ATTCATATAATTTGATTACATTTAAAATTCCTGATAACCCAAATTCCTAAGAG-ATAACCTAAGTAATATTCAACCATAATAAATAGTTTCTTCATG 797

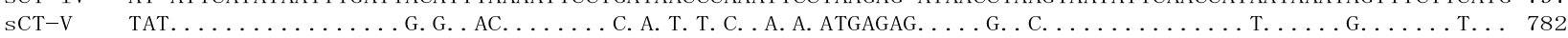

SCT-IV CCTCACCATGTTTCTTGTTCAATAAACCTATTTTTCTACC---

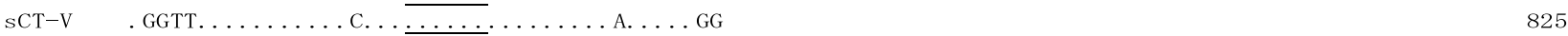

Figure 1 Nucleotide sequences of cDNAs encoding SCTs-IV and -V from the rainbow trout. The coding region for sCT precursors is indicated by grey boxes; a black box specifies the sCT-encoding sequences. Gaps marked by hyphens (-) are inserted to optimize homology. Dots represent nucleotides identical to those of sCT-IV cDNA. Nucleotides are numbered from $5^{\prime}$ to $3^{\prime}$, beginning with the first base in the coding region. The AATAAA polyadenylation signals are underlined.

coding for sCTs-I and -IV were determined by sequence analysis. The sCT-I-encoding fragment was then used as a probe to screen 18000 recombinants from the rainbow trout cDNA library. About 1080 positive CT clones were obtained from the rainbow trout, and sequence analysis revealed the complete nucleotide sequences of two distinct sCT cDNAs: one for sCT-IV and the other for a novel sCT, which we have designated as sCT-V (Figs 1 and 2), in addition to the previously isolated sCT-I cDNAs (AB094135, AB094136). No cDNA clones were obtained for sCTs-II or -III.

\section{Rainbow trout SCT cDNA and precursor}

The cDNAs for rainbow trout sCT-IV (AB094137) and $-\mathrm{V}$ (AB094138) precursors consisted of 925 and
915 bases respectively, excluding the poly(A) tail (Fig. 1). Both the precursors were predicted to encode a signal peptide of 25 amino acid residues, an N-terminal procalcitonin (proCT), a putative cleavage site Lys-Arg, calcitonin, a cleavage and amidation sequence Gly-Lys-Lys-Arg, and a C-terminal peptide of 18 amino acids (Fig. 2). The rainbow trout ${ }_{S} C T s-I V$ and $-\mathrm{V}$ conserved a sequence for potential N-linked glycosylation (Asn88-Leu89-Ser90), although the biological significance of CT glycosylation has yet to be determined (Fig. 2). The sCT-V was identical to sCT-IV except for Met at position 8, and the sCT-V precursor exhibited a $90 \%$ identity with the sCT-IV precursor. A phylogenetic tree of CT precursors from the rainbow trout, chum salmon, puffer fish, chicken, mouse, rat and human was created using the Clustal W algorithm (Fig. 3). In 
Rainbow trout sCT-IV

Rainbow trout sCT-V

Rainbow trout sCT-Ia

Rainbow trout $\mathrm{sCT}-\mathrm{Ib}$

Chum salmon CT-I

Fugu CT

Chicken CT

Mouse CT

Rat CT

Human CT

Rainbow trout sCT-IV Rainbow trout $\mathrm{sCT}-\mathrm{V}$ Rainbow trout sCT-Ia Rainbow trout sCT-Ib Chum salmon CT-I

Fugu CT

Chicken CT

Mouse CT

Rat CT

Human CT
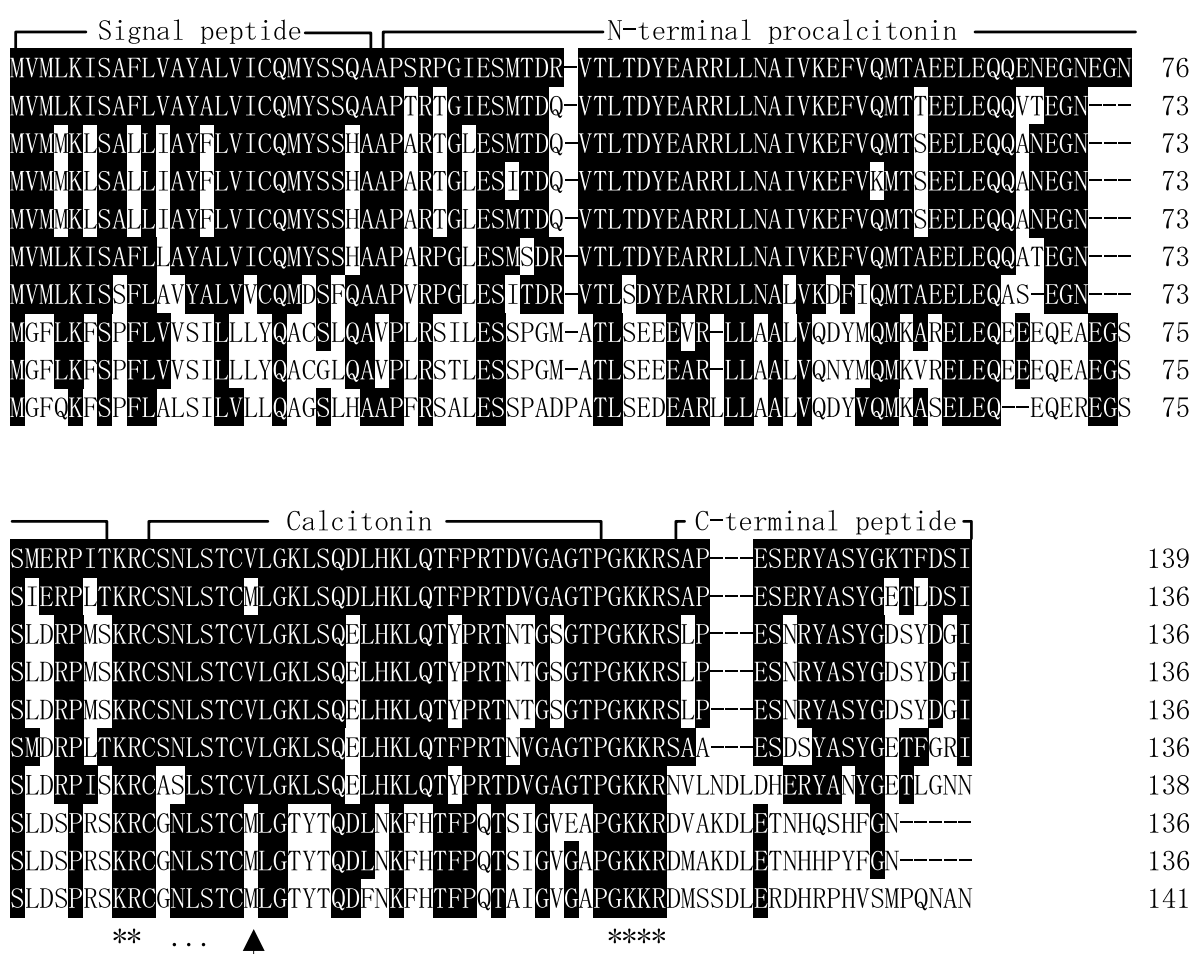

139

136

136

136

136

136

138

136

136

141

Figure 2 Comparison of amino acid sequences among the CT precursors of rainbow trout (accession numbers AB094135-AB094138), chum salmon (Y00765), puffer fish (Fugu; AJ309015), chicken (X03012), mouse (X97991), rat (V01228) and human (X00356). The sequences were aligned using Clustal W (Thompson et al. 1994). Gaps marked by hyphens (-) have been inserted to optimize homology. Conserved amino acids are shown in black boxes. The SCT-V is a novel peptide with Val of SCT-IV substituted by Met (arrow). The sCT-V precursor is most similar to the sCT-IV precursor, with $90 \%$ identity. The sCT precursors are $43-52 \%$ identical to mammalian CT precursors. Asterisks denote potential signals for proteolytic processing, and dots indicate a sequence for potential $\mathrm{N}$-linked glycosylation.

this tree, the chum salmon sCT-I precursor is linked with the rainbow trout $\mathrm{sCT}-\mathrm{Ia}$ and $-\mathrm{Ib}$ precursors, whereas the rainbow trout sCT-IV and -V precursors form another cluster. The tree also indicates that the rainbow trout $\mathrm{sCT}$ precursors are much more closely related to chicken CT precursors than to mammalian counterparts. The topology of this phylogenetic tree is consistent with the similarity among CT peptides from these species (Sasayama 1999, Suzuki et al. 1999b), and further suggests that the CT genes of teleost and chicken might be more similar than is estimated by phylogenetic analysis of CT regions (Clark et al. 2002).

\section{Tissue distribution of sCT mRNAs}

Calcitonin gene expression in rainbow trout tissues was first investigated by RT-PCR using the oligonucleotide primers that can amplify all the known sCT-encoding sequences. A cDNA band of the predicted length was observed in all the tissues examined, including the ultimobranchial gland (Fig. 4a). In contrast, RT-PCR analysis of CT mRNA in rat tissues indicated that rat CT gene expression was restricted to the thyroid containing CT-secreting C cells, brain, stomach, lung, adipose tissue, testis, ovary and kidney (Fig. 4b). Southern blot analysis using CT cDNA probes from the rainbow trout or rat confirmed the authenticity of those amplified bands (data not shown).

Tissue distribution of sCT-I, -IV and -V mRNAs was further examined in the rainbow trout by RT-PCR using specific primers. The specificity of primers for each of the sCT cDNAs had been assessed by performing PCR with the sCT clones as templates. Although sCT-IV and -V cDNAs had sequences very similar to those of the rtCT-I 


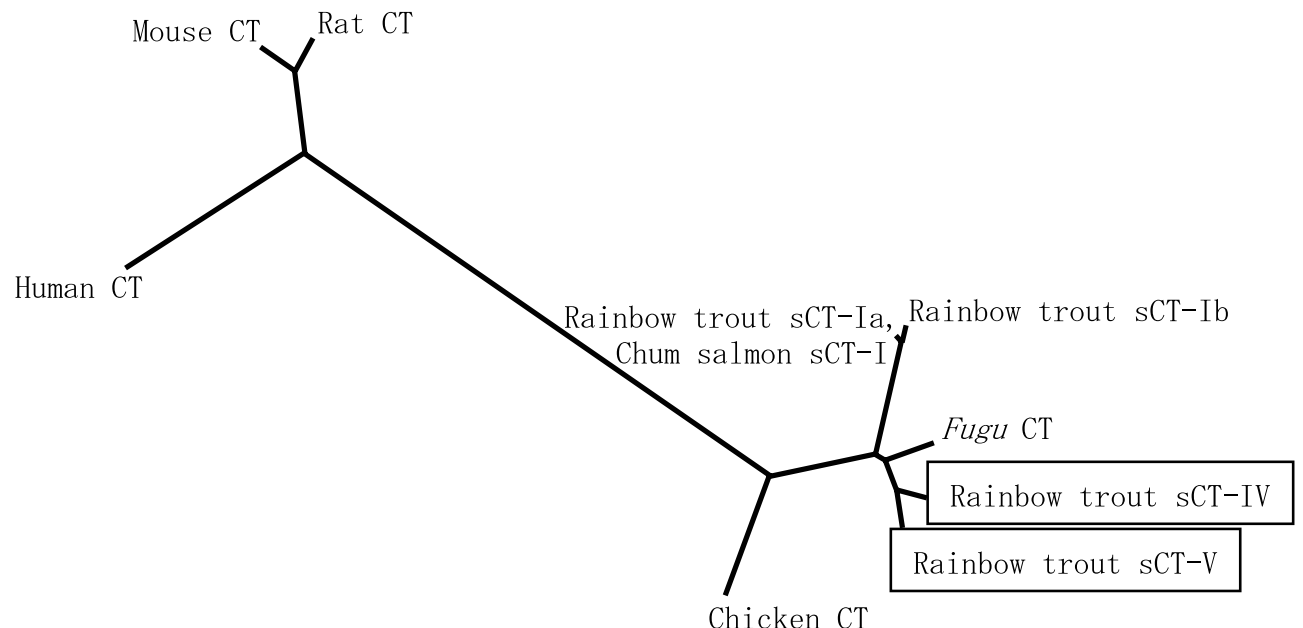

Figure 3 Phylogenetic tree of CT precursors from the rainbow trout, chum salmon, puffer fish (Fugu), chicken, mouse, rat and human, obtained by the neighbour-joining method (Saitou \& Nei 1987). The length of each branch is proportional to the mean number of differences per residue. The topology suggests that the $s C T-V$ gene might have diverged from the lineage to $s C T-I V$ gene by a gene duplication event. The fish CT precursors are more closely related to chicken CT precursors than to their mammalian counterparts.

primers (Table 1), these primers amplified only sCT-I cDNA (Fig. 5a, lanes 1-3). It was verified that the amplified sCT-I cDNA fragment was cut with a restriction enzyme, PstI (Fig. 5a, lane 4), as predicted by sequence analysis. PCR with the rtCT-IV primers (Table 1) amplified sCT-V cDNA in addition to sCT-IV (Fig. 5b, lanes 2, 3). However, the PGR amplicon from sCT-IV cDNA was shown to be cut by another restriction enzyme, NspI (Fig. 5b, lane 4), indicating that the amplification of sCT-IV cDNA can be distinguished from that of ${ }_{s C T-V}$, and can be confirmed by this treatment. The rtCT-V primers (Table 1) amplified only the corresponding cDNA fragment (Fig. 5c), as they were synthesized on the basis of the sequence of insertion unique to $\mathrm{SCT}-\mathrm{V}$ cDNA.

RT-PCR analysis for rainbow trout tissues using the rtCT-I primers, coupled with PstI digestion, revealed that $\mathrm{sCT}$-I mRNA was expressed, not only in the ultimobranchial gland, but also in the testis, ovary and gills (Fig. 6). After NspI treatment of the PCR bands amplified with rtCT-IV primers, expression of sCT-IV mRNA was found in the testis in addition to the ultimobranchial gland (Fig. 6). A PGR band for sCT-IV mRNA was also detected in the pituitary, but the authenticity of this band could not be verified because the amounts of amplification products were insufficient for restric- tion digestion. Although the amino acid sequence of sCT-V peptide was nearly the same as that of sCT-IV (Fig. 2), the distribution of sCT-V mRNA was unique and was detected in most tissues examined, except the kidney, gills and blood cells (Fig. 6). In spite of the wide distribution of the sCT mRNAs, all were expressed in the greatest amounts in the ultimobranchial gland, as clearly shown by the quantification analysis (Fig. 6). RT-PCR examination further revealed that the sCT-I, -IV and $-\mathrm{V}$ mRNAs were all expressed in the ultimobranchial gland of a single trout (data not shown). The PCR amplicons from these sCT mRNAs were also observed in a rainbow trout of a different strain (data not shown), corroborating the existence of $s C T-I,-I V$ and $-V$ genes in the genome of this species.

\section{Histological localization of SCTs-IV and -V mRNAs}

The sites of sCT-IV and -V mRNA expressions were examined by in situ hybridization histochemistry with DIG-labelled antisense RNA probes. Hybridization signal for both sCT-IV (Fig. 7a) and sCT-V (Fig. 7c) mRNA was observed abundantly in the parenchymal cells of trout ultimobranchial gland. The sCT-IV mRNA was also detected at low levels in the Leydig cells of the testis (Fig. 7b). 

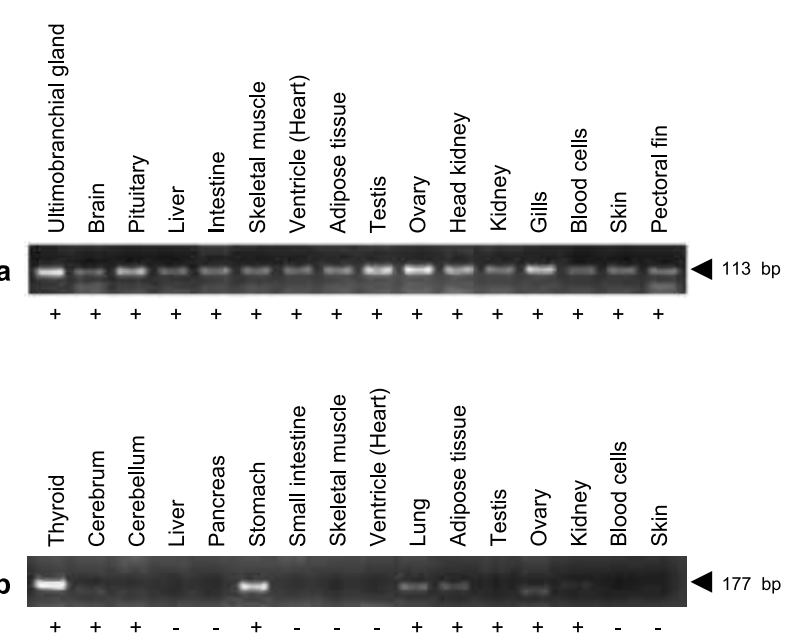

Figure 4 Tissue distribution of CT mRNA in (a) rainbow trout and (b) rat, determined by RT-PCR analysis. Total RNA $(10 \mu \mathrm{g})$ from various tissues were reversetranscribed and amplified by PCR with specific oligonucleotide primers: SCT primers for rainbow trout and $\mathrm{mCT}$ primers for rat (Table 1). Compared with the other samples, a tenfold dilution of the PCR products from the ultimobranchial gland was electrophoresed in (a), because of the abundant expression of SCT mRNA in this site. The presence of amplified bands at the predicted lengths (113 bp for rainbow trout, and $177 \mathrm{bp}$ for rat) shows that SCT mRNA was expressed in all the trout tissues examined (a), whereas expression of CT mRNA was limited to several tissues in rat (b).

Although RT-PGR analysis indicated the presence of sCT-V mRNA in various tissues (Fig. 6), we could not detect any distinct signals in their wax sections (data not shown), suggesting much lower expression levels of $\mathrm{sCT}-\mathrm{V}$ mRNA in these tissues.

Figure $\mathbf{5}$ Specificity of pairs of PCR primers used to examine the expression of (a) $s C T-I$, (b) $s C T$ - $I V$ and (c) $s C T$-V genes. PCR amplification with rtCT-I, -IV and -V primers (Table 1) was performed for each of the pBK-CMV phagemid vectors containing SCT-I (lane 1), sCT-IV (lane 2), or SCT-V (lane 3) cDNAs (0.4 ng). The rtCT-I and -V primers were specific for SCTs-I and -V respectively, and the SCT-I amplicons were accordingly cleaved into two fragments of the predicted lengths (104 bp and $66 \mathrm{bp}$ ) by restriction digestion with Pstl (a, lane 4). The rtCT-IV primers amplified not only SCT-IV cDNA (b, lane 2), but also sCT-V cDNA (b, lane 3); however, the presence of SCT-IV cDNA could be detected by digestion of the PCR products with Nspl, generating three fragments of the predicted lengths (180 bp, $116 \mathrm{bp}$ and $46 \mathrm{bp}$ ) (b, lane 4).

\section{Discussion}

In the present study, two distinct cDNAs encoding sCT-IV and a novel sCT-V were cloned from the ultimobranchial gland of rainbow trout. The initiation site for translation was predicted to be
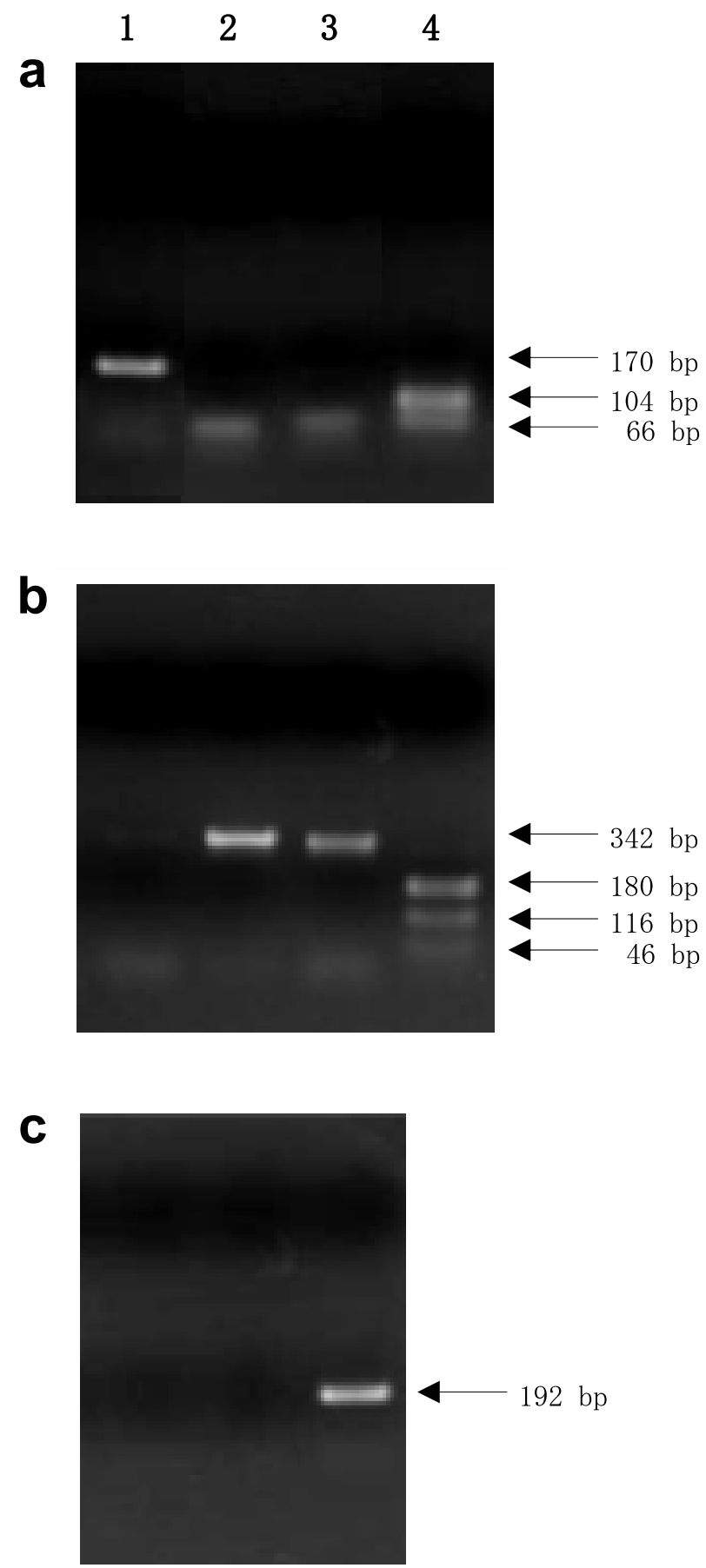

Journal of Molecular Endocrinology (2004) 32, 963-974

www.endocrinology.org 


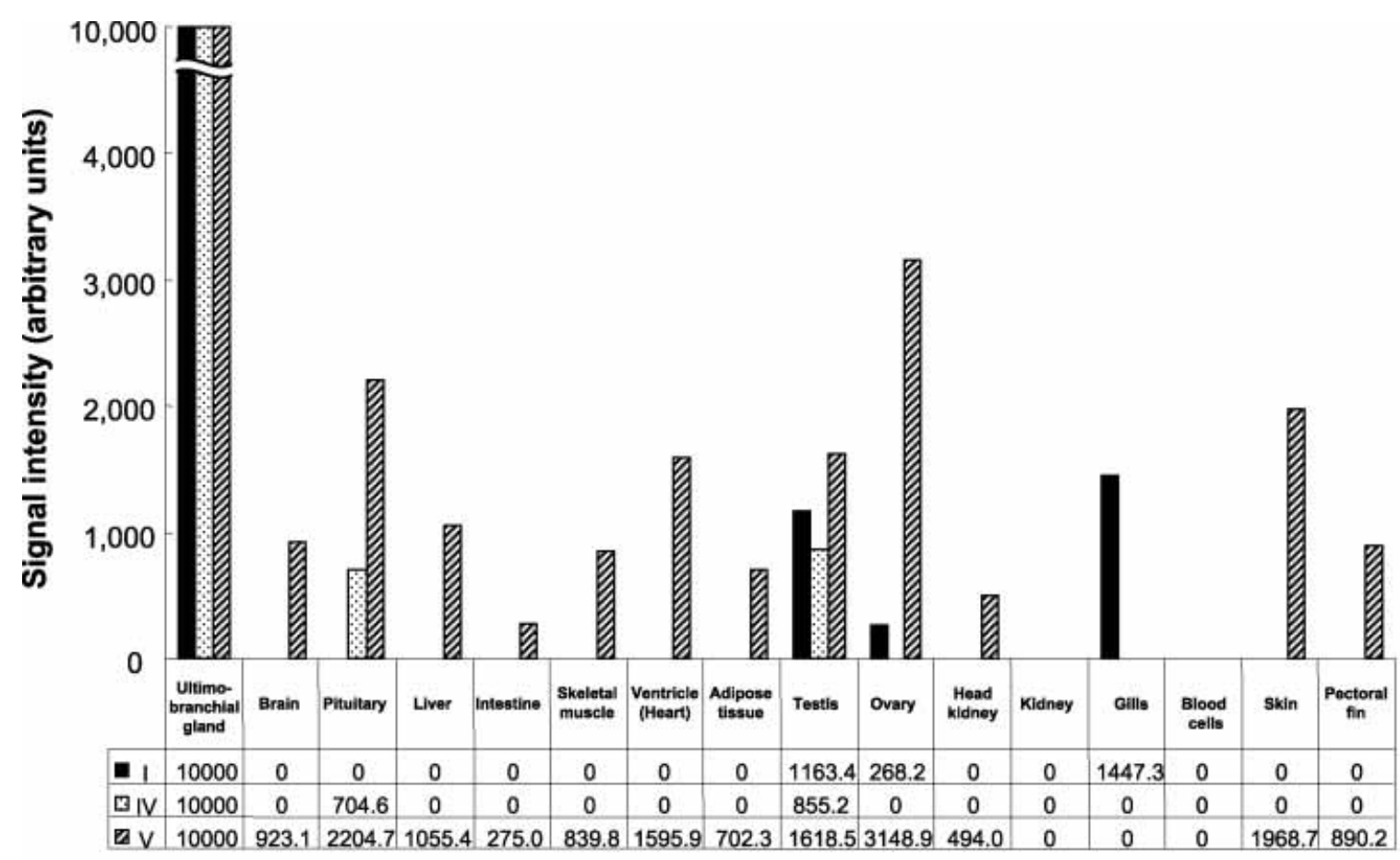

SCT-I $\rightleftharpoons-\leftarrow$ bp

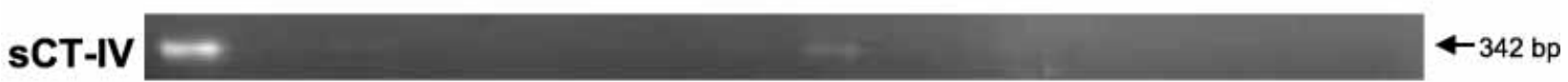

SCT-V -

\section{EF1- $\alpha$}

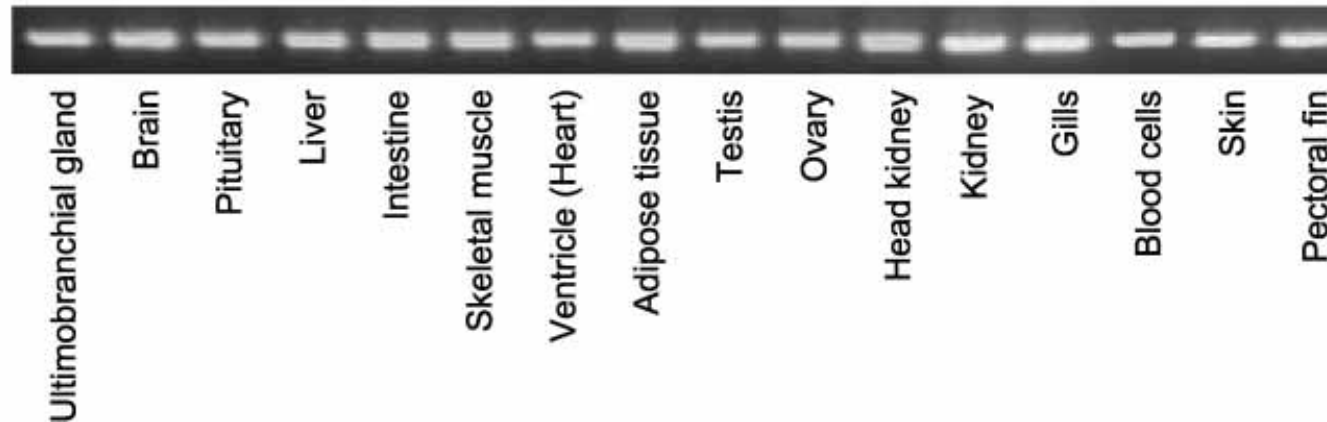

Figure 6 Tissue distribution of SCT-I, -IV and -V mRNAs in the rainbow trout, determined by RT-PCR analysis. Total RNA $(10 \mu \mathrm{g})$ from various trout tissues were reverse-transcribed and amplified by PCR with rtCT-I, -IV and -V primers (Table 1). Distinct bands for SCT-I mRNA were detected in the ultimobranchial gland, gonads and gills, whereas a prominent band for SCT-IV mRNA was seen for the testis and ultimobranchial gland. The authenticity of these bands was confirmed by specific digestion with restriction enzymes: Pstl for sCT-I, and Nspl for sCT-IV. In contrast, the expression of SCT-V mRNA was shown to occur in most tissues examined, but not in the blood cells. The relative levels of mRNA expression were normalized to EF1- $\alpha$ (upper panel), with the maximum value of each sCT defined as 10000 . 

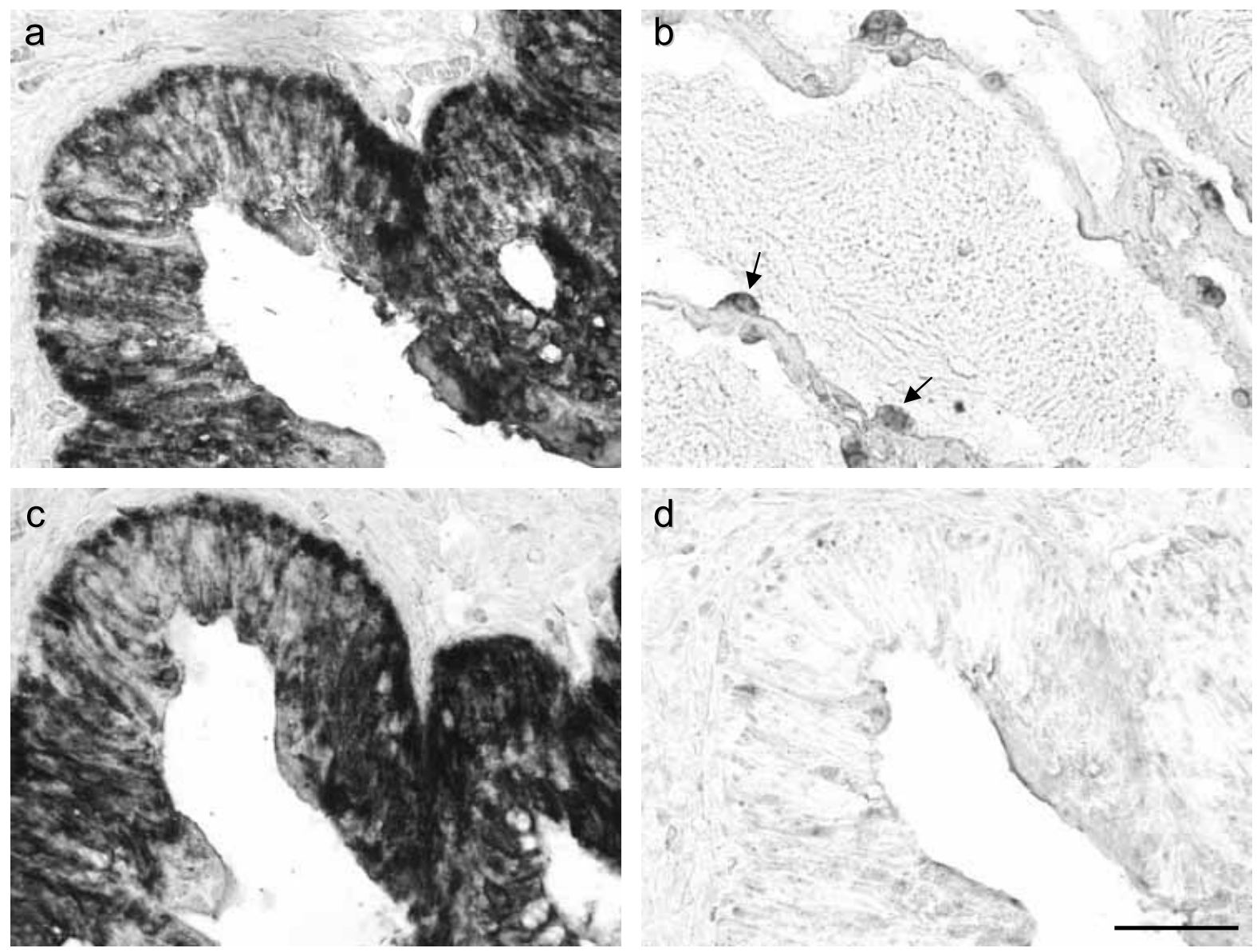

Figure 7 In situ hybridization histochemistry for (a, b) sCT-IV and (c) sCT-V mRNAs in wax sections (6 $\mu \mathrm{m})$ of trout tissues. (a) Parenchymal cells of the ultimobranchial gland showed an intense hybridization signal with the DIG-labelled antisense SCT-IV cRNA probe. (b) A weak signal for SCT-IV mRNA was also seen in the Leydig cells (arrows) of the testis. (c) A strong signal for SCT-V transcripts was evident throughout the ultimobranchial gland. (d) Control section for (c). Hybridization with the sense cRNA probe exhibited no signal on the ultimobranchial gland. Scale bar represents $50 \mu \mathrm{m}$.

ATG at positions $1-3$ in each cDNA (Fig. 1), because the sequence ACCATGG at positions -3 to 4 was identical with the consensus sequence for initiation sites in other eukaryotic genes (Kozak 1981). The deduced sCT-IV and $-\mathrm{V}$ precursors were composed of an N-terminal peptide, sCT and a C-terminal peptide, but the N-terminal peptide was predicted to be further cleaved into a signal peptide of 25 amino acids and an N-terminal proCT, by analogy with other teleost N-terminal peptides (Suzuki et al. 1998) and human preproCT (Russwurm et al. 1999). In view of the presence of sCT-I cDNAs (AB094135, AB094136), our sequence analysis suggests that three types of $\mathrm{CT}$, i.e. sCTs-I, -IV and $-\mathrm{V}$, could be produced in the rainbow trout. The RT-PGR analysis and in situ hybridization histochemistry further indicated strong expression of their mRNAs in the ultimobranchial gland, suggesting that sCTs-I, -IV and -V are all secreted into the circulation from this site. It was also shown by the RT-PGR that the sCT mRNAs were expressed in various tissues at lower levels. It has been reported that the mRNA encoding a possible CT receptor is present in a number of tissues from the flounder, such as the gills, brain, intestine, testis and ovary (Suzuki et al. 2000b). It has been also documented that CT receptor mRNAs are expressed in most mammalian tissues (Kuestner et al. 1994, Sexton et al. 1999). Therefore, receptors for sCT are likely to 
exist also in those trout tissues in which sCT-I, -IV or $-\mathrm{V}$ mRNAs were detected, implying an autocrine or paracrine action (or both) of sCT.

As for mammals, there is evidence to suggest that CT has various physiological functions in addition to preventing hypercalcaemia and encouraging bone mineralization (Sexton et al. 1999). For instance, Nakhla et al. (1989) indicated that CT could regulate testicular function because $\mathrm{sCT}$ stimulated the secretion of androgen-binding protein from primary Sertoli cell-enriched cultures and increased the concentrations of both androgen and oestrogen receptors in TM4 cells that were derived from immature mouse Sertoli cells. In fish, CT-immunoreactive cells have been observed in the intestine of goldfish, and the population of these cells increased in response to a soup lacking calcium more than to calcium-enriched soup, although the plasma calcium concentration was greatest in goldfish fed with the latter (Okuda et al. 1999). It is therefore proposed that CT cells in the intestine might affect the absorption of nutrients in this fish. With respect to salmonid fish, little is known about the local functions of sCT. However, our RT-PCR analysis has demonstrated the tissue distribution of sCT-I, -IV and V mRNAs in various trout tissues. Intriguingly, their patterns of tissue expression were remarkably different: sCT-IV mRNA was detected in the testis, whereas sCT-V mRNA occurred in most tissues examined. Expression of CT mRNA had already been reported in the gills of pink salmon and rainbow trout (Martial et al. 1994, Sasayama 1999), but our RT-PCR analysis revealed that the CT mRNA expressed in this tissue is only sCT-I. In addition, in situ hybridization histochemistry showed expression of $s C T-I V$ gene in the Leydig cells of trout testis. The cells expressing sCT-V mRNA could not be localized in any tissues other than the ultimobrachial gland by this technique, but it has been the experience of many laboratories that in situ hybridization can be ineffectual in detecting the sites of expression of transcripts when their levels of expression are low (Nelhs et al. 1996, Jagger et al. 2000). Furthermore, an investigation using radioimmunoassay demonstrated lower concentrations of CT-like immunoreactivity in various tissues of the rainbow trout (Fouchereau-Peron et al. 1990), in some of which only sCT-V mRNA was detected by our RT-PGR analysis. Therefore, sCT-V mRNA seems to be expressed at very low levels in most tissues, as suggested by the RT-PCR. Taken together, our results suggest that sCTs-I, -IV and -V might be produced locally in a tissue-specific manner. Although there is no direct evidence, these sCTs might have different local functions, depending on their particular tissue distribution.

Much attention has been focused on proCT, because it is a specific marker for systemic inflammatory responses in humans (Russwurm et al. 1999, Whicher et al. 2001). It has also been reported that injection of proCT augments the mortality of hamsters suffering from sepsis (Nylen et al. 1998). It would be of great interest to examine whether pro-sCTs have similar effects in rainbow trout, even though the amino acid homology between fish and mammalian proCTs is less than that between their CTs.

Structural comparison with other CTs indicates that $\mathrm{sCT}-\mathrm{V}$ is a new peptide in which the Val at position 8 of sCTs-I and -IV is replaced by Met. It is known that sCT-IV inhibits bone-slice resorption in a pit formation assay, and shows an even greater hypocalcaemic potency than sCT-I in a rat bioassay (Schecroun et al. 1999). Because rat, rabbit and human CTs have Met at position 8, as occurs in ${ }_{s C T} \mathrm{~V},{ }_{\mathrm{sCT}} \mathrm{V}$ could prove to have some biological activities that might be used for the therapeutic treatment of bone disorders.

The present phylogenetic analysis of CT precursors demonstrates that rainbow trout $\mathrm{SCT}$ genes can be classified into two groups: one containing $s C T-I a$ and $-I b$ genes, and the other $s C T-I V$ and $-V$ genes. As rainbow trout is tetraploid (Ohno et al. 1968, Hiraoka et al. 1993), these two groups of $s C T$ genes might have been generated by a genome duplication event in ancestral fish, and thereafter $s C T-I V$ and $-V$ genes might have diverged by gene duplication. In addition, it is possible that other $s C T$ genes may exist in the genome of rainbow trout, because sCT-I, -IV and -V mRNAs were not detected in the kidney and blood cells in which sCT cDNAs were amplified by PCR using sCT primers corresponding to the conserved sequences flanking the sCT-coding region (Fig. 4a).

The RT-PGR analysis of the tissue distribution of sCT mRNAs showed that sCT-I, -IV and -V mRNAs were all expressed most abundantly in the ultimobranchial gland of rainbow trout. Nothing, however, is known about the molecular mechanisms that intensify $s C T$ gene expression in this 
site. Our elucidation of the sCT cDNAs has now made it possible to identify the entire $s C T$ genes of the trout and determine whether homologous enhancer elements occur in the 5'-flanking DNA of these $s C T$ genes. In mammals, luciferase reporter assays with HeLa and 44-2C cell lines revealed that an 18 bp element (5'-GGGAGCTGTGCAAA TCCT), located approximately $1 \mathrm{kbp}$ upstream of the transcriptional initiation site of rat CT gene, has a strong enhancer activity (Tverberg \& Russo 1993). A sequence nearly identical to this element is also present in the human CT/CGRP enhancer region (Broad et al. 1989, Peleg et al. 1990). These distal enhancers contain potential binding elements for helix-loop-helix (HLH) protein and octamerbinding protein designated OB2. It has been suggested that the major HLH complex can be a heterodimer of the ubiquitous upstream stimulatory factors-1 and -2 (Lanigan \& Russo 1997). Although OB2 protein has not yet been cloned, mobility shift competition assays indicate the existence of this factor in the CT/CGRP-producing CA77 and 44-2C cells, but not in non-C cell lines such as HeLa, GH3, and Rat-1, suggesting that OB2 protein is a cell-specific activator. Therefore, OB2-like protein might exist in the ultimobranchial gland of rainbow trout, enhancing $s C T$ gene expression.

\section{Acknowledgements}

We are grateful to the staff of Fuji Trout Hatchery for the supply of rainbow trout, and to the staff of the Fuji Branch of Shizuoka Prefectural Fisheries Experimental Station for their help in sampling. We express our sincere gratitude to Prof. Susumu Hyodo and Sanae Hasegawa, University of Tokyo, Japan, for their assistance in this study, to Prof. Bridget Baker for critical reading of the manuscript, and to Prof. Shigeyasu Tanaka, Shizuoka University, Japan, for his encouragement. This work was supported by Grants-in-Aid from the Ministry of Education, Science, Sports, and Culture of Japan, and in part by funds from the cooperative programme (No. 12 2002) provided by Ocean Research Institute, University of Tokyo.

\section{References}

Becker LK, Snider HR, Moore FC, Monaghan GK \& Silva LO 1979 Calcitonin in extrathyroidal tissues of man. Acta Endocrinologica 92 746-751.
Bjornsson BT, Haux C, Forlin L \& Deftos LJ 1986 The involvement of calcitonin in the reproductive physiology of the rainbow trout. Fournal of Endocrinology 108 17-23.

Broad MP, Symes JA, Thakker VR \& Craig KR 1989 Structure and methylation of the human calcitonin/alpha-CGRP gene. Nucleic Acid Research 17 6999-7011.

Clark SM, Bendell L, Power MD, Warner S, Elgar G \& Ingleton MP 2002 Calcitonin: characterisation and expression in a teleost fish, Fugu rubripes. Fournal of Molecular Endocrinology 28 111-123.

Craig KR, Hall L, Edbrooke RM, Allison J \& MacIntyre I 1982 Partial nucleotide sequence of human calcitonin precursor mRNA identifies flanking cryptic peptides. Nature 295 345-347.

Ding QY, Zhu JL, Bagchi KM \& Bagchi CI 1994 Progesterone stimulates calcitonin gene expression in the uterus during implantation. Endocrinology 135 2265-2274.

Fouchereau-Peron M, Arlot-Bonnemains Y, Taboulet J, Milhaud G \& Moukhtar SM 1990 Distribution of calcitonin gene-related peptide and calcitonin-like immunoreactivity in trout. Regulatory Peptides 27 171-179.

Hidaka Y, Tanaka S \& Suzuki M 2004 Analysis of salmon calcitonin $\mathrm{I}$ in the ultimobranchial gland and gillfilaments during development of rainbow trout Oncorhynchus mykiss, by in situ hybridization and immunochemical staining. Zoological Science. In press.

Hiraoka S, Suzuki M, Yanagisawa T, Iwata M \& Urano A 1993 Divergence of gene expression in neurohypophysial hormone precursors among salmonids. General and Comparative Endocrinology 92 292-301.

Jacobs WJ, Goodman HR, Chin WW, Dee CP \& Habener FJ 1981 Calcitonin messenger RMA encodes multiple polypeptides in a single precursor. Science 213 457-459.

Jagger C, Chambers T \& Pondel M 2000 Transgenic mice reveal sites of calcitonin receptor gene expression during development. Biochemical and Biophysical Research Communications 274 124-129.

Jansz H, Martial K, Zandberg J, Milhaud G, Benson AA, Julienne A, Moukhtar MS \& Cressent M 1996 Identification of a new calcitonin gene in the salmon Oncorhynchus gorbuscha. Proceedings of the National Academy of Sciences of the USA 93 12344-12348.

Kozak M 1981 Possible role of flanking nucleotides in recognition of the AUG initiator codon by eukaryotic ribosomes. Nucleic Acids Research 9 5233-5252.

Kuestner ER, Elrod DR, Grant JF, Hagen SF, Kuijper LJ, Matthewes LS, O'Hara JP, Sheppard OP, Stroop DS, Thompson LD et al. 1994 Cloning and characterization of an abundant subtype of the human calcitonin receptor. Molecular Pharmacology 46 246-255.

Lanigan MT \& Russo FA 1997 Binding of upstream stimulatory factor and a cell-specific activator to the calcitonin/calcitonin gene-related peptide enhancer. Fournal of Biological Chemistry 272 18316-18324

Lasmoles F, Jullienne A, Day F, Minvielle S, Milhaud G \& Moukhtar MS 1985 Elucidation of the nucleotide sequence of chicken calcitonin mRNA: direct evidence for the expression of a lower vertebrate calcitonin-like gene in man and rat. EMBO fournal 4 2603-2607.

Martial K, Maubras L, Taboulet J, Jullienne A, Milhaud G, Moukhtar SM \& Cressent M 1994 Production of salmon calcitonin I in Oncorhynchus gorbuscha by alternative polyadenylation of two RNA species. Gene 149 277-281.

Maubras L, Cressent M, Minvielle S, Taboulet J, Jullienne A, Milhaud G \& Moukhtar SM 1990 Expression of the calcitonin gene during migration of the Pacific salmon, Oncorhynchus gorbuscha. Biochemical and Biophysical Research Communications 173 788-794.

Nakhla AM, Mather JP, Janne OA \& Bardin CW 1989 The action of calcitonin on the $\mathrm{TM}_{4}$ Sertoli cell line and on rat Sertoli cell-enriched culture. Fournal of Andrology 10 321-331.

Nehls M, Kyewski B, Messerle M, Waldschutz R, Schuddekopf K, 
Smith AJH \& Boehm T 1996 Two genetically separable steps in the differentiation of thymic epithelium. Science $\mathbf{2 7 2}$ 886-889.

Nylen SE, Whang TK, Snider HR, Steinwald MP, White CJ \& Becker LK 1998 Motality is increased by procalcitonin and decreased by an antiserum reactive to procalcitonin in experimental sepsis. Critical Care Medicine 26 1001-1006.

Ohno S, Wolf U \& Atkin NB 1968 Evolution from fish to mammals by gene duplication. Hereditas $\mathbf{5 9} 169-187$.

Okuda R, Sasayama Y, Suzuki N, Kambegawa A \& Srivastav KA 1999 Calcitonin cells in the intestine of goldfish and a comparison of the number of cells among saline-fed, soup-fed or high Ca soup-fed fishes. General and Comparative Endocrinology 113 267-273.

Peleg S, Abruzzese VR, Cote JG \& Gagel FR 1990 Transcription of the human calcitonin gene is mediated by a $\mathrm{C}$ cell-specific enhancer containing E-box-like elements. Molecular Endocrinology 4 $1750-1757$.

Poeschl E, Lindley I, Hofer E, Seifert JM, Brunowsky W \& Besemer J 1987 The structure of procalcitonin of the salmon as deduced from its cDNA sequence. FEBS Letters 226 96-100.

Rehli M, Luger K, Beier W \& Falk W 1996 Molecular cloning and expression of mouse procalcitonin. Biochemical and Biophysical Research Communications 226 420-425.

Russwurm S, Wiederhold M, Oberhoffer M, Stonans I, Zipfel FP \& Reinhart K 1999 Molecular aspects and natural source of procalcitonin. Clinical Chemistry and Laboratory Medicine 37 789-797.

Saitou N \& Nei M 1987 The neighbor-joining method: a new method for reconstructing phylogenetic trees. Molecular Biology and Evolution 4 406-425.

Sasayama Y 1999 Hormonal control of Ca homeostasis in lower vertebrates: considering the evolution. Zoological Sciences $\mathbf{1 6}$ 857-869.

Schecroun N, Pidoux E, Horne W, Hernandez-Lagunas L, Baron R \& Cressent M 1999 Biological properties of salmon calcitonin IV. Fournal of Bone and Mineral Research 14 1425-1431.

Sexton MP, Findlay MD \& Martin JT 1999 Calcitonin. Current Medical Chemistry 6 1067-1093.

Sheward JW, Lutz ME \& Harmar JA 1994 The expression of the calcitonin receptor gene in the brain and pituitary gland of the rat. Neuroscience Letters 181 31-34.

Suzuki M, Hyodo S, Kobayashi M, Aida K \& Urano A 1992 Characterization and localization of mRNA encoding the salmon-type gonadotrophin-releasing hormone precursor of the masu salmon. Fournal of Molecular Endocrinology 9 73-82.

Suzuki M, Fujikura K, Inagaki N, Seino S \& Takata K 1997 Localization of the ATP-sensitive $\mathrm{K}^{+}$channel subunit Kir6.2 in mouse pancreas. Diabetes 46 1440-1444.

Suzuki N, Nishizawa T, Kase Y, Sasayama Y, Takei Y, Kaiya H, Ukawa K, Noso T, Kambegawa A \& Srivastav K 1998 Amino acid sequences of $\mathrm{N}$-terminal procalcitonin of some vertebrates. Netherlands Fournal of Zoology 48 349-360.

Suzuki N, Suzuki D, Sasayama Y, Srivastav KA, Kambegawa A \& Asahina K 1999a Plasma calcium and calcitonin levels in eels fed a high calcium solution or transferred to seawater. General and Comparative Endocrinology 114 324-329.

Suzuki N, Ueda K, Sakamoto H \& Sasayama Y 1999b Fish calcitonin genes: primitive bony fish genes have been conserved in some lower vertebrates. General and Comparative Endocrinology 113 369-373.

Suzuki N, Suzuki T \& Kurokawa T 2000a Suppression of osteoclastic activities by calcitonin in the scales of goldfish (freshwater teleost) and nibbler fish (seawater teleost). Peptides 21 115-124.

Suzuki N, Suzuki T \& Kurokawa T $2000 b$ Cloning of a calcitonin gene-related peptide receptor and a novel calcitonin receptor-like receptor from the gill of flounder, Paralichthys olivaceus. Gene $\mathbf{2 4 4}$ 81-88.

Thompson JD, Higgins DG \& Gibson TJ 1994 CLUSTAL W: improving the sensitivity of progressive multiple sequence alignment through sequence weighting, position-specific gap penalties and weight matrix choice. Nucleic Acids Research 22 4673-4680.

Tverberg AL \& Russo FA 1993 Regulation of the calcitonin/calcitonin-gene-related peptide gene by cell-specific synergy between helix-loop-helix and octamer-binding transcription factors. Fournal of Biological Chemistry $\mathbf{2 6 8}$ 15965-15973.

Whicher J, Bienvenu J \& Monneret G 2001 Procalcitonin as an acute phase marker. Annals of Clinical Biochemistry 38 483-493.

\section{Received in final form 3 November 2003 Accepted 6 February 2004}

